\title{
Risk factors for infection following prostate biopsy - a case control study
}

Elliot Anderson ${ }^{{ }^{*}}$, Olivia Leahy ${ }^{2}$, Allen C. Cheng ${ }^{3,4}$ and Jeremy Grummet ${ }^{2,5}$

\begin{abstract}
Background: Infection is a complication of TRUS prostate biopsy, despite the use of antimicrobial prophylaxis. Worryingly the rate of infectious complications following TRUS biopsy has been shown to be increasing. We aimed to determine the rate, severity, risk factors, standard patterns of care and microbiology resistance profiles associated with TRUS biopsy sepsis.

Methods: A retrospective case-control study was conducted. Using electronic coding all patients who presented to Cabrini Hospital with sepsis following a TRUS biopsy from 2009 to 2013 were identified. Validated cases were matched to controls in a ratio of 1:3. Eligible controls were required to have undergone a TRUS biopsy at the same surgical institution as the case and in the closest period of time. Demographic, procedural and patient related data-points were recorded for all patients using hospital and urologist records. Univariate logistic regression models were constructed and used to determine risk factors associated with infection.

Results: 71 cases developed sepsis following TRUS biopsy and were matched to 213 controls. The average rate of sepsis over the 5 -year study period was $1.5 \%$. A SOFA score $\geq 2$ was identified in $28 \%$ of cases. We found a high prevalence of antimicrobial resistant E. coli, with $61 \%$ of blood culture isolates classified as Multidrug resistant organisms. Eight different prophylactic antimicrobial regimens were identified with $33 \%$ of cases receiving ineffective antimicrobial prophylaxis. Statistically significant risk factors included previous antimicrobial use and prior international travel within the six months prior to biopsy.

Conclusions: TRUS biopsy is an elective procedure and as such needs to be associated with minimal morbidity. The patterns of care surrounding periprocedural variables for TRUS biopsies were non-uniform and diverse. A wide variety of different prophylaxis regimens and bowel preparation routines were recorded. Patients with risk factors for sepsis may represent a better target population for intervention with alternative preventative strategies. Alternative preventative options include augmented prophylaxis, tailored prophylaxis or the TP biopsy approach either as a first line biopsy modality or based on epidemiological risk factors.
\end{abstract}

Keywords: Prostate, Needle biopsy, Transrectal biopsy, Infection, Sepsis, Case control

\section{Background}

The diagnosis of prostate cancer is confirmed by prostate biopsy. Transrectal ultrasound-guided (TRUS) biopsy is the most common method of obtaining prostate tissue for analysis [1]. TRUS biopsies are typically considered to be a well-tolerated outpatient procedure however complications are well recognized [2]. Current practice guidelines unanimously recommend the use of antimicrobial prophylaxis prior to a TRUS biopsy,

\footnotetext{
* Correspondence: elliotpeteranderson@gmail.com

'Monash University, Melbourne, Victoria, Australia

Full list of author information is available at the end of the article
}

which has been shown to significantly decrease the risk of post-biopsy infectious complications [3-5]. However infectious complications may still develop with the reported rate of sepsis ranging from 0.6-5.7 \% in contemporary studies $[6,7]$ with evidence of an increasing trend $[8,9]$.

The proposed pathophysiology of post-biopsy infection most likely results from the transrectal passage of the biopsy needle. This approach allows for the inoculation of bacteria from the rectal mucosa directly into the prostate, blood vessels or urinary tract $[10,11]$. The best evidence suggests that the presence of endogenous antimicrobial- 
resistant bacteria is a significant risk factor for infectious complications [12-15]. In particular, Steensels et al. found that the presence of endogenous fluoroquinolone-resistant bacteria in men undergoing a TRUS biopsy was a risk factor for developing post-biopsy infectious complications [16]. The risk of acquiring endogenous antimicrobialresistant bacteria may be increased by several factors such as antimicrobial use [17-20] or international travel prior to TRUS biopsy [20, 21].

Other risk factors for developing sepsis following TRUS biopsy can be categorized as patient-related or procedural. No definitive patient or procedure-specific risk factors have been identified, despite analysis of patient co-morbidities [9, 22, 23], the use of pre-biopsy enema $[6,24]$ or the number of cores taken during the procedure [25].

In the present study we aimed to determine the rate, severity, risk factors and microbiology resistance patterns associated with TRUS biopsy sepsis. As well as the standard patterns of care surrounding TRUS prostate biopsy.

\section{Methods}

The population was defined as patients undergoing a TRUS prostate biopsy by a urologist associated with Cabrini Hospital, a private 700-bed metropolitan hospital in Melbourne. This included patients who had their TRUS biopsy at smaller neighbouring institutions, but in the event of complications were instructed to present to Cabrini Hospital's Emergency Department. Neighbouring institutions included Linacre Private Hospital, Masada Private Hospital and the Avenue Hospital. All biopsies were completed using a standard ultrasound and biopsy probe with patients receiving prophylactic antimicrobials and intravenous sedation prior to their procedure. A case was defined as a patient who presented to hospital with sepsis due to suspected or confirmed infection related to the genitourinary tract or where no other focus of infection was clinically evident within 14 days of undergoing a TRUS biopsy from 2009 until 2013. Sepsis was defined according to the ACCP/SCCM (1991) criteria as a systemic inflammatory response syndrome in the presence of an infective process [26]. Patients were excluded if they underwent TRUS biopsy at a non-participating institution. Cases were identified via an electronic search of the Cabrini Health data warehouse using Medicare Benefit Schedule (MBS) codes for TRUS biopsy and International Classification of Disease and Related Health Problems, Tenth Revision, Australian Modification (ICD-10-AM) diagnosis codes for infective complications. These codes were used to screen for sepsis, which was then confirmed by a review of the patients' medical records. Confirmed cases that underwent their TRUS biopsy at a participating institution were matched to three control patients. Eligible controls were the next patients that underwent TRUS biopsy at the same institution as the case but whom did not develop infectious complications.

Patient data, including demographic, procedural and patient-related factors were collected on a standardized form using hospital and urologist records. Where the procedure was performed at another institution, procedural data was extracted from urologists' medical records. A history of travel was defined as international travel within six months prior to biopsy. Antimicrobial use was defined as oral or intravenous antimicrobial therapy within the six months preceding biopsy. If no history was documented, it was assumed the patient had not travelled or taken antimicrobials. We used a modified Sequential Organ Failure Assessment Score (SOFA) to determine the severity of illness. The SOFA score is a standardised assessment tool used to determine the degree of sepsis related organ dysfunction. It is calculated from the total sum of six organ system categories, each graded from 0 to 4 based on predefined measurement values. As many patients did not have an arterial blood gas, we used arterial oxygen saturation measured by a pulse oximeter $(\mathrm{SpO} 2)$ as a surrogate marker for partial pressure of arterial oxygen $\left(\mathrm{PaO}_{2}\right)$ [27].

To define risk factors associated with infection, we constructed univariate conditional logistic regression models, which accounted for the matched nature of the control selection. A multiple variable analysis was not performed due to the relatively low proportion of patients with any one exposure (and therefore limited statistical power) and the likelihood of co-linearity between exposures. We defined statistical significance as $p<0.05$. As this was an exploratory study, we did not adjust for multiple hypothesis testing. Ethics approval was obtained from the Cabrini Human Research Ethics Committee.

\section{Results}

Over the five-year study period, 71 patients required admission to Cabrini Hospital for sepsis following TRUS biopsy and were matched to 219 controls. The mean proportion of patients with sepsis across the five-year study period was $1.5 \%$, with the lowest rate of $0.6 \%$ reported in 2011 and the highest rate of $2.9 \%$ reported in 2013. No statistically significant difference regarding the rate of sepsis over the study period was noted ( $p=0.091)$.

The mean duration of admission was 4.5 days (median 4 days, IQR 3,5 days, range 1-14 days). Of the 60 (76.9\%) cases where data was available to assess a SOFA score, $43(71.7 \%)$ patients had a SOFA score $\leq 1,10$ $(16.7 \%)$ patients had a score of two, 4 (6.7 \%) had a SOFA score of three and 3 (5\%) patients had a SOFA score $\geq 4$. 
Urine culture results were available for 66 patients. Culture results were positive for 28 (42.4\%) patients. Isolated organisms included 27 Escherichia coli (96.4 \%) and one Acinetobacter spp. (3.6\%). Of all urine isolates, $22(78.6 \%)$ were resistant to more than one antimicrobial agent. Blood culture results were available for 65 patients. Culture results were positive for 36 (55.4\%) patients. The organisms isolated were E. coli 34 (94.4 \%), Streptococcus pneumoniae 1(2.9\%) and Bacteroides uniformis 1(2.9\%). Of all blood isolates 28 (77.7 \%) were resistant to more than one antimicrobial agent (Table 1).

Patient risk factors that were found to be significantly associated with the development of sepsis following TRUS biopsy included a history of international travel or recent antimicrobial use in the six months prior to a patient's biopsy (Table 2). Other variables such as patient comorbidities, recent hospitalization, age or being a hospital employee were not significantly associated with sepsis.

The number of biopsy cores most commonly taken was 14 [Range: 7,22]. More than 12 cores were taken in $63(88.7 \%)$ case cohort patients and in 202 (94.8\%) of control cohort patients. Patients who did not develop sepsis were significantly more likely to have had more than 12 biopsy cores taken (OR 0.37, 95 \% CI 0.14, 0.95,

Table 1 Antimicrobial resistance in breakthrough blood/urine isolates

\begin{tabular}{|c|c|c|c|}
\hline $\begin{array}{l}\text { Antimicrobial } \\
\text { class }\end{array}$ & Antimicrobial name & $\begin{array}{l}\text { Urine } \\
\text { culture (\%) }\end{array}$ & $\begin{array}{l}\text { Blood } \\
\text { culture (\%) }\end{array}$ \\
\hline \multirow[t]{2}{*}{ Aminoglycosides } & Gentamicin & $15(53.6)$ & $20(58.8)$ \\
\hline & Tobramycin & $3(10.7)$ & $14(41.2)$ \\
\hline \multirow[t]{3}{*}{ Cephalosporins } & Cefepime & 0 & $4(11.7)$ \\
\hline & Ceftriaxone & $4(14.3)$ & $6(17.6)$ \\
\hline & $\begin{array}{l}\text { Cephalexin or } \\
\text { Cephazolin }\end{array}$ & $8(28.6)$ & $12(35.2)$ \\
\hline Carbapenems & Imipenem & 0 & 0 \\
\hline Fluoroquinolone & $\begin{array}{l}\text { Ciprofloxacin or } \\
\text { Norfloxacin }\end{array}$ & $22(78.6)$ & $23(67.6)$ \\
\hline Nitrofurans & Nitrofurantoin & $2(7.1)$ & 0 \\
\hline \multirow[t]{4}{*}{ Penicillins } & $\begin{array}{l}\text { Ampicillin or } \\
\text { Amoxycillin }\end{array}$ & $18(64.3)$ & $24(70.6)$ \\
\hline & $\begin{array}{l}\text { Amoxycillin/clavulanic } \\
\text { acid }\end{array}$ & $8(28.6)$ & $20(58.8)$ \\
\hline & Piperacillin & $3(10.7)$ & $10(29.4)$ \\
\hline & Ticarcillin/clavulanate & $6(21.4)$ & $8(23.5)$ \\
\hline \multirow[t]{3}{*}{ Sulfonamides } & Trimethoprim & $17(60.7)$ & $2(5.9)$ \\
\hline & $\begin{array}{l}\text { Trimethoprim/ } \\
\text { sulfamethoxazole }\end{array}$ & $6(21.4)$ & $11(32.4)$ \\
\hline & Total isolate organisms ${ }^{a}$ & 28 & 34 \\
\hline
\end{tabular}

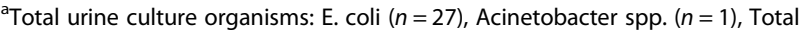
blood culture organisms: E. coli $(n=34)$ $p=0.04)$. Other procedural variables such as the use of a pre-biopsy enema, prior TRUS biopsy, prostate volume or biopsy result were not shown to be significantly associated with infection.

All patients in this study received antimicrobial prophylaxis. Over the course of the study eight different antimicrobial regimens were identified (Table 3). Seven of the identified regimens included the use of a fluoroquinolone antimicrobial and this accounted for 282 $(99.3 \%)$ men. The most common prophylaxis regimen was the use of a fluoroquinolone agent alone, followed by a fluoroquinolone antimicrobial in combination with a penicillin antimicrobial. All prophylactic regimens were distributed equally across the study period. This study found that the addition of a penicillin antimicrobial (amoxicillin) to standard fluoroquinolone prophylaxis resulted in a reduced risk of developing sepsis (OR 0.42, $95 \%$ CI 0.18, 0.98, $p=0.05)$. However, when this risk was calculated without carbapenem co-administration, $26.8 \%$ of cases and $31.5 \%$ of controls developed sepsis suggesting pencillins were not effective. There was also shown to be no significant difference between the use of three days or seven days of fluoroquinolone antimicrobials (OR 1.72, $95 \%$ CI 0.71, 4.20, $p=0.23$ ) No patient who received a carbapenem antimicrobial prophylactically developed sepsis following a TRUS biopsy.

\section{Discussion}

In this study, we found that the rate of post-biopsy sepsis was $1.5 \%$ across the five-year study period. This is consistent with the intervention arms of the original clinical trials supporting prophylaxis [4], and may reflect relatively low rates of fluoroquinolone resistance in Australia [28]. This is in contrast to studies in North America that have demonstrated a rising rate of postbiopsy sepsis most likely attributable to increasing bacterial resistance $[8,9]$. In addition we found that a third of septic patients had evidence of significant organ dysfunction, consistent with severe sepsis, although we did not observe any mortality. This is in line with previous studies suggesting the mortality from prostate biopsy is low $[8,9]$.

As in similar studies, E. coli was the predominant causative organism in our series, accounting for $95 \%$ of positive cultures $[13,25,29,30]$. A concerning trend has been the development and spread of genes providing resistance to beta-lactams, quinolones and aminoglycoside antimicrobials [31]. Fluoroquinolone resistance has been linked to failure of prophylaxis in previous studies [16, 17]. Notably in our series, $68 \%$ and $59 \%$ of breakthrough blood culture isolates were resistant to fluoroquinolones and gentamicin respectively. However, we found that only a third of cases received an ineffective prophylaxis regimen, based on resistance patterns of 
Table 2 Risk factors for sepsis

\begin{tabular}{|c|c|c|c|c|}
\hline & Cases & Controls & OR $(95 \%$ Cl) & $P$ value \\
\hline Number & 71 & 213 & & \\
\hline \multicolumn{5}{|l|}{ Patient variables } \\
\hline Age $>65$ years & $35(49.3 \%)$ & $92(43.2 \%)$ & $1.34(0.74,2.40)$ & 0.33 \\
\hline Hospital employee & $5(7.0 \%)$ & $13(6.1 \%)$ & $1.16(0.41,3.33)$ & 0.78 \\
\hline Autoimmune condition & $0(0 \%)$ & $10(4.5 \%)$ & Undefined & ND \\
\hline Immunosuppression & $1(1.4 \%)$ & $3(1.4 \%)$ & $1.00(0.08,11.93)$ & 1.00 \\
\hline COPD & $2(2.8 \%)$ & $4(1.9 \%$ & $1.50(0.27,8.19)$ & 0.64 \\
\hline Heart valve replacement & $3(4.2 \%)$ & $3(1.4 \%)$ & $3.0(0.61,14.86)$ & 0.18 \\
\hline Benign prostate enlargement & $14(19.7 \%)$ & $41(19.2)$ & $1.24(0.62,2.43)$ & 0.54 \\
\hline Diabetes & $10(14.1 \%)$ & $23(10.8 \%)$ & $1.35(0.61,2.96)$ & 0.46 \\
\hline Recent travel & $13(18.3 \%)$ & $7(3.2 \%)$ & $5.40(1.61,18.09)$ & 0.01 \\
\hline Recent antimicrobial use & $7(9.8 \%)$ & $3(1.4 \%)$ & $9.59(1.97,46.62)$ & 0.01 \\
\hline Recent hospitalisation & $1(1.4 \%)$ & $0(0.0 \%)$ & Undefined & ND \\
\hline \multicolumn{5}{|l|}{ Procedural variables } \\
\hline Use of FQ prophylaxis & $70(98.6 \%)$ & $213(100.0 \%)$ & Undefined & ND \\
\hline Duration of FQ prophylaxis $<7$ days & $10(14.1 \%)$ & $44(20.7 \%)$ & $1.72(0.71,4.20)$ & 0.23 \\
\hline Use of aminoglycoside prophylaxis & $19(26.8 \%)$ & $43(20.2 \%)$ & $1.85(0.81,4.24)$ & 0.14 \\
\hline Use of a penicillin as prophylaxis & $19(26.8 \%)$ & $76(35.7 \%)$ & $0.42(0.18,0.98)$ & 0.05 \\
\hline Use of carbapenem as prophylaxis & 0 & $21(9.8 \%)$ & Undefined & ND \\
\hline Previous biopsy & $12(16.9 \%)$ & $36(16.9 \%)$ & $1.29(0.62,2.70)$ & 0.50 \\
\hline Previous biopsy within 3 years & $11(15.5 \%)$ & $26(12.2 \%)$ & $1.31(0.61,2.83)$ & 0.48 \\
\hline Urinary catheter & $0(0 \%)$ & $0(0 \%)$ & Undefined & ND \\
\hline Preoperative urine culture & $1(1.4 \%)$ & $0(0 \%)$ & Undefined & ND \\
\hline Use of enema & $31(43.6 \%)$ & $110(51.6 \%)$ & $0.71(0.30,1.69)$ & 0.44 \\
\hline Prostate volume $>30 \mathrm{~mL}$ & $53(74.6 \%)$ & $174(81.7 \%)$ & $1.38(0.64,2.93)$ & 0.41 \\
\hline $\mathrm{PSA}>4 \mathrm{mmol}$ & $55(77.4 \%)$ & $168(78.8 \%)$ & Undefined & ND \\
\hline Number of biopsy cores $>12$ & $46(64.8 \%)$ & 156 (73.2 \%) & $0.37(0.14,0.95)$ & 0.04 \\
\hline Prostate cancer & 42 (59.2 \%) & 123 (57.7 \%) & $1.15(0.63,2.07)$ & 0.65 \\
\hline
\end{tabular}

ND not done

Table 3 Prophylactic antimicrobial regimens used

\begin{tabular}{lll}
\hline${\text { Antimicrobial } \text { class }^{\mathrm{a}}}^{\mathrm{a}}$ & \# Cases (\%) & \# Controls (\%) \\
\hline $\mathrm{F}$ & $28(39.4)$ & $88(41.3)$ \\
$\mathrm{F}+\mathrm{P}$ & $18(25.3)$ & $63(29.5)$ \\
$\mathrm{F}+\mathrm{P}+\mathrm{A}$ & $1(1.4)$ & $4(1.8)$ \\
$\mathrm{F}+\mathrm{A}$ & $23(32.3)$ & $37(17.3)$ \\
$\mathrm{F}+\mathrm{P}+\mathrm{C}$ & $0(0)$ & $8(3.7)$ \\
$\mathrm{F}+\mathrm{P}+\mathrm{A}+\mathrm{C}$ & $0(0)$ & $1(0.4)$ \\
$\mathrm{F}+\mathrm{C}$ & $0(0)$ & $11(5.1)$ \\
$\mathrm{C}+\mathrm{A}+\mathrm{CE}$ & $0(0)$ & $1(0.4)$ \\
Unknown & $1(1.4)$ & $0(0)$ \\
Total & 71 & 213 \\
\hline
\end{tabular}

${ }^{\mathrm{a}} F$ fluoroquinolone, $P$ penicillin, $A$ aminoglycoside, $C$ carbapenem, $C E$ cephalosporin isolated organisms. This suggests that factors other than antimicrobial resistance may be responsible for the failure of prophylaxis, and that switching from fluoroquinolones to aminoglycosides may not necessarily be more effective. The proportion with fluoroquinolone resistance is similar to that found in a New Zealand study [32] but lower than in North American studies [14].

A wide diversity of antimicrobial prophylaxis regimens has been reported in the literature [33-37]. This study identified eight different prophylaxis protocols over the study period reflecting considerable clinical uncertainty. The most effective prophylactic regimens include carbapenem antimicrobials with this study supporting Losco et al's finding of a $0 \%$ sepsis rate amongst men undergoing a TRUS biopsy [38]. Although carbapenem antimicrobials appear to be highly effective, they can only be administered 
parenterally, and there is concern that widespread use may result in the development and spread of carbapenemresistant Enterobacteriaceae (CRE) [38, 39]. Other strategies to minimize risk include the use of alternative prophylactic agents (e.g. nitrofurantoin and/or fosfomycin), $[40,41]$ the use of targeted prophylaxis based on microbiological screening for resistance, or alternative surgical approaches, such as transperineal biopsy.

Consistent with other reports, we found that international travel in the six months prior to the procedure was associated with post-biopsy sepsis. Although this association may be over-estimated due to recall bias, it is known that the prevalence of fluoroquinoloneresistance organisms varies around the world [28], that travel is associated with carriage of antimicrobial resistant bowel flora [21] and that travel to countries with high fluoroquinolone-resistance has been shown to be a risk factor for developing sepsis following TRUS biopsy $[20,42]$. Interestingly, we found that all patients who developed sepsis and travelled internationally had journeyed to the South-East Asia or Western Pacific regions. This may suggest that the risk profile of the destination may be useful in assessing risk, and help identify a group where alternative strategies or antimicrobial agents may be warranted.

This study had a number of limitations. In this observational study, we cannot exclude the possibility of unmeasured confounders. This was particularly the case as a large number of different prophylaxis regimens were used, with little or no documentation about the rationale for the choices of agents. We attempted to minimize selection bias by selecting cases and controls from the same population in a systematic manner. Recall bias may be present due to cases being more thoroughly questioned about risk factors than controls. This was true for travel history, with recorded responses for $51 \%$ of cases and $16 \%$ of controls. Conversely patients were more thoroughly questioned about recent antimicrobial use, with recorded responses for $73 \%$ of cases and $92 \%$ of controls. It is possible that patients who underwent TRUS biopsy at participating hospitals presented with infections elsewhere, although routine advice was given to patients to represent to Cabrini Hospital if complications developed as it is the only private hospital in this region with an emergency department. In addition the use of coding to identify cases is subject to inherent error in data capture and relevant patients may have consequently been missed. The absence of positive clinical isolates for all septic patients reflects the sensitivity of cultures as well as the use of prior antibiotics. The association between a higher number of cores and reduced sepsis is difficult to explain and is likely to reflect confounding by unknown factors.

\section{Conclusion}

In this study TRUS biopsy sepsis was found to inflict a significant impact on patients and the healthcare system. A wide variety of different prophylaxis regimens and bowel preparation routines were recorded. Procedural variables such as extended prophylaxis regimens and the use of an enema did not show added benefit and may therefore be superfluous. This study also identified recent international travel and recent antimicrobial use as risk factors that were associated with sepsis. Patients with these factors may represent a better target population for intervention with alternative preventative strategies. These options include augmented prophylaxis, tailored prophylaxis or the transperineal biopy approach.

\section{Competing interests}

The authors declare that they have no competing interests. AC is supported by a Australian NHMRC Career Development Fellowship.

\section{Authors' contributions}

$\mathrm{EA}, \mathrm{OL}, \mathrm{AC}$ and JG participated in the conception and design of the study and analysis and interpretation of data. EA and OL were involved in the acquisition of data. AC performed the statistical analysis. All authors helped to draft the manuscript and have read and approved the final manuscript.

\section{Acknowledgements}

The authors would like to thank David Phillips and Cabrini Health, particularly Cabrini Health Information Services for their assistance.

\section{Author details}

${ }^{1}$ Monash University, Melbourne, Victoria, Australia. ${ }^{2}$ Department of Urology, Alfred Health, Melbourne, Victoria, Australia. ${ }^{3}$ Department of Epidemiology and Preventative Medicine, Monash University, Melbourne, Victoria, Australia. ${ }^{4}$ Department of Infectious Diseases, Alfred Health, Melbourne, Victoria, Australia. ${ }^{5}$ Department of Surgery, Monash University, Melbourne, Victoria, Australia.

Received: 29 April 2015 Accepted: 17 December 2015

Published online: 23 December 2015

\section{References}

1. Heidenreich A, Bellmunt J, Bolla M, Joniau S, Mason M, Matveev V, et al. EAU guidelines on prostate cancer. Part 1: screening, diagnosis, and treatment of clinically localised disease. Eur Urol. 2011;59(1):61-71.

2. Challacombe B, Dasgupta P, Patel U, Amoroso P, Kirby R. Recognizing and managing the complications of prostate biopsy. BJU Int. 2011;108(8):1233-4.

3. Kapoor DA, Klimberg IW, Malek GH, Wegenke JD, Cox CE, Patterson AL, et al. Single-dose oral ciprofloxacin versus placebo for prophylaxis during transrectal prostate biopsy. Urol. 1998;52(4):552-8.

4. Aron M, Rajeev TP, Gupta NP. Antibiotic prophylaxis for transrectal needle biopsy of the prostate: a randomized controlled study. BJU Int. 2000;85(6):682-5.

5. Puig J, Darnell A, Bermudez P, Malet A, Serrate G, Bare M, et al. Transrectal ultrasound-guided prostate biopsy: is antibiotic prophylaxis necessary? Eur Radiol. 2006;16(4):939-43.

6. Zaytoun OM, Anil T, Moussa AS, Jianbo L, Fareed K, Jones JS. Morbidity of prostate biopsy after simplified versus complex preparation protocols: assessment of risk factors. Urol. 2011;77(4):910-4.

7. Pace G, Carmignani L, Marenghi C, Mombelli G, Bozzini G. Cephalosporins periprostatic injection: are really effective on infections following prostate biopsy? Int Urol Nephrol. 2012:44(4):1065-70.

8. Nam RK, Saskin R, Lee Y, Liu Y, Law C, Klotz LH, et al. Increasing hospital admission rates for urological complications after transrectal ultrasound guided prostate biopsy. J Urol. 2010;183(3):963-8.

9. Loeb S, Carter HB, Berndt SI, Ricker W, Schaeffer EM. Complications after prostate biopsy: data from SEER-Medicare. J Urol. 2011;186(5):1830-4. 
10. Ozden E, Bostanci Y, Yakupoglu KY, Akdeniz E, Yilmaz AF, Tulek N, et al. Incidence of acute prostatitis caused by extended-spectrum betalactamase-producing Escherichia coli after transrectal prostate biopsy. Urol. 2009;74(1):119-23.

11. Liss MA, Chang A, Santos R, Nakama-Peeples A, Peterson EM, Osann K, et al. Prevalence and significance of fluoroquinolone resistant Escherichia coli in patients undergoing transrectal ultrasound guided prostate needle biopsy. J Urol. 2011;185(4):1283-8.

12. Batura D, Rao GG, Nielsen PB. Prevalence of antimicrobial resistance in intestinal flora of patients undergoing prostatic biopsy: implications for prophylaxis and treatment of infections after biopsy. BJU Int. 2010; 106(7):1017-20.

13. Horcajada JP, Busto M, Grau S, Sorli L, Terradas R, Salvado M, et al. High prevalence of extended-spectrum beta-lactamase-producing enterobacteriaceae in bacteremia after transrectal ultrasound-guided prostate biopsy: a need for changing preventive protocol. Urol. 2009;74(6):1195-9.

14. Lange D, Zappavigna C, Hamidizadeh R, Goldenberg SL, Paterson RF, Chew $\mathrm{BH}$. Bacterial sepsis after prostate biopsy-a new perspective. Urol. 2009; 74(6):1200-5.

15. Taylor AK, Zembower TR, Nadler RB, Scheetz MH, Cashy JP, Bowen D, et al. Targeted antimicrobial prophylaxis using rectal swab cultures in men undergoing transrectal ultrasound guided prostate biopsy is associated with reduced incidence of postoperative infectious complications and cost of care. J Urol. 2012;187(4):1275-9.

16. Steensels D, Slabbaert K, De Wever L, Vermeersch P, Van Poppel H, Verhaegen J. Fluoroquinolone-resistant E. coli in intestinal flora of patients undergoing transrectal ultrasound-guided prostate biopsy—should we reassess our practices for antibiotic prophylaxis? Clin Microbiol Infect. 2012;18:575-81.

17. Mosharafa AA, Torky MH, El Said WM, Meshref A. Rising incidence of acute prostatitis following prostate biopsy: fluoroquinolone resistance and exposure is a significant risk factor. Urol. 2011;78(3):511-4.

18. Akduman B, Akduman D, Tokgoz H, Erol B, Turker T, Ayoglu F, et al. Longterm fluoroquinolone use before the prostate biopsy may increase the risk of sepsis caused by resistant microorganisms. Urol. 2011;78(2):250-5.

19. Abughosh Z, Margolick J, Goldenberg SL, Taylor SA, Afshar K, Bell R, et al. A prospective randomized trial of povidone-iodine prophylactic cleansing of the rectum before transrectal ultrasound guided prostate biopsy. J Urol. 2013;189(4):1326-31.

20. Patel U, Dasgupta P, Amoroso P, Challacombe B, Pilcher J, Kirby R. Infection after transrectal ultrasonography-guided prostate biopsy: increased relative risks after recent international travel or antibiotic use. BJU Int. 2012;109(12):1781-5.

21. Kennedy K, Collignon P. Colonisation with Escherichia coli resistant to "critically important" antibiotics: a high risk for international travellers. Eur J Clin Microbiol Infect Dis. 2010;29(12):1501-6.

22. Ehdaie B, Vertosick E, Spaliviero M, Giallo-Uvino A, Taur Y, O'Sullivan M, et al. The impact of repeat biopsies on infectious complications in men with prostate cancer on active surveillance. J Urol. 2014;191(3):660-4.

23. Pinkhasov Gl, Lin YK, Palmerola R, Smith P, Mahon F, Kaag MG, et al. Complications following prostate needle biopsy requiring hospital admission or emergency department visits - experience from 1000 consecutive cases. BJU Int. 2012;110(3):369-74.

24. Jeon SS, Woo S-H, Hyun J-H, Choi HY, Chai SE. Bisacodyl rectal preparation can decrease infectious complications of transrectal ultrasound-guided prostate biopsy. Urol. 2003;62(3):461-6.

25. Carignan A, Roussy JF, Lapointe V, Valiquette L, Sabbagh R, Pepin J. Increasing risk of infectious complications after transrectal ultrasoundguided prostate biopsies: time to reassess antimicrobial prophylaxis? Eur Urol. 2012;62(3):453-9.

26. Levy MM, Fink MP, Marshall JC, Abraham E, Angus D, Cook D, et al. 2001 SCCM/ESICM/ACCP/ATS/SIS International Sepsis Definitions Conference. Crit Care Med. 2003;31(4):1250-6.

27. Pandharipande PP, Shintani AK, Hagerman HE, St Jacques PJ, Rice TW, Sanders NW, et al. Derivation and validation of Spo2/Fio2 ratio to impute for Pao2/Fio2 ratio in the respiratory component of the sequential organ failure assessment score. Crit Care Med. 2009;37(4):1317-21.

28. Cheng AC, Turnidge J, Collignon P, Looke D, Barton M, Gottlieb T. Control of fluoroquinolone resistance through successful regulation, Australia. Emerg Infect Dis. 2012;18(9):1453-60.
29. Simsir A, Kismali E, Mammadov R, Gunaydin G, Cal C. Is it possible to predict sepsis, the most serious complication in prostate biopsy? Urol Int. 2010; 84(4):395-9.

30. Loeb S, van den Heuvel S, Zhu X, Bangma CH, Schroder FH, Roobol MJ. Infectious complications and hospital admissions after prostate biopsy in a European randomized trial. Eur Urol. 2012;61(6):1110-4.

31. Cagnacci S, Gualco L, Debbia E, Schito GC, Marchese A. European emergence of ciprofloxacin-resistant Escherichia coli clonal groups O25:H4ST 131 and 015:K52:H1 causing community-acquired uncomplicated cystitis. J Clin Microbiol. 2008;46(8):2605-12.

32. Williamson DA, Roberts SA, Paterson DL, Sidjabat H, Silvey A, Masters J, et al. Escherichia coli bloodstream infection after transrectal ultrasound-guided prostate biopsy: implications of fluoroquinolone-resistant sequence type 131 as a major causative pathogen. Clin Infect Dis. 2012;54(10):1406-12.

33. Lee G, Attar K, Laniado M, Karim O. Trans-rectal ultrasound guided biopsy of the prostate: nationwide diversity in practice and training in the United Kingdom. Int Urol Nephrol. 2007;39(1):185-8.

34. Webb NR, Woo HH. Antibiotic prophylaxis for prostate biopsy. BJU Int. 2002; 89(8):824-8.

35. Shandera K: Variability in patient preparation for prostate biopsy among American Urologists. 1998

36. Davis M, Sofer M, Kim SS, Soloway MS. The procedure of transrectal ultrasound guided biopsy of the prostate: a survey of patient preparation and biopsy technique. J Urol. 2002;167(2 Pt 1):566-70.

37. Taylor HM, Bingham JB. Antibiotic prophylaxis for transrectal prostate biopsy. J Antimicrob Chemother. 1997;39(2):115-7.

38. Losco G, Studd R, Blackmore T. Ertapenem prophylaxis reduces sepsis after transrectal biopsy of the prostate. BJU Int. 2014;113 Suppl 2:69-72.

39. Zaytoun OM, Vargo EH, Rajan R, Berglund R, Gordon S, Jones JS. Emergence of fluoroquinolone-resistant Escherichia coli as cause of postprostate biopsy infection: implications for prophylaxis and treatment. Urol. 2011;77(5):1035-41.

40. Lipsky BA, Byren I, Hoey CT. Treatment of bacterial prostatitis. Clin Infect Dis. 2010;50(12):1641-52.

41. Gardiner BJ, Mahony AA, Ellis AG, Lawrentschuk N, Bolton DM, Zeglinski PT, et al. Is fosfomycin a potential treatment alternative for multidrug-resistant gram-negative prostatitis? Clin Infect Dis. 2014;58(4):e101-105.

42. Williamson DA, Masters J, Freeman J, Roberts S. Travel-associated extendedspectrum beta-lactamase-producing Escherichia coli bloodstream infection following transrectal ultrasound-guided prostate biopsy. BJU Int. 2012; 109(7):E21-22

\section{Submit your next manuscript to BioMed Central and we will help you at every step:}

- We accept pre-submission inquiries

- Our selector tool helps you to find the most relevant journal

- We provide round the clock customer support

- Convenient online submission

- Thorough peer review

- Inclusion in PubMed and all major indexing services

- Maximum visibility for your research

Submit your manuscript at www.biomedcentral.com/submit
C Biomed Central 